\title{
Topological structure evolvement of flow and temperature fields in deformable drop Marangoni migration in microgravity
}

\author{
Jian-Fu Zhao a,*, Liang Zhang ${ }^{\text {a }}$, Zhen-Dong Li ${ }^{\mathrm{b}}$, Wen-Tao Qin ${ }^{\mathrm{c}}$ \\ ${ }^{a}$ Key Laboratory of Microgravity (National Microgravity Laboratory)/CAS, Institute of Mechanics, Chinese Academy of Sciences (CAS), Beijing 100190, China \\ ${ }^{\mathrm{b}}$ State Nuclear Power Technology Research \& Development Center, Beijing 100190, China \\ ${ }^{\mathrm{c}}$ Henan Puyang Electric Power Company, Puyang 457000, China
}

\section{A R T I C L E I N F O}

\section{Article history:}

Received 24 March 2011

Received in revised form 2 June 2011

Available online 25 June 2011

\section{Keywords:}

Thermocapillary migration

Topological structure

Liquid drop

\begin{abstract}
A B S T R A C T
Using the level-set method and the continuum interface model, the axisymmetric thermocapillary migration of a deformable liquid drop immerged in an immiscible bulk liquid with a temperature gradient is simulated numerically with constant material properties of the two phases. Steady terminal state of the motion can always be reached. The dimensionless terminal migration velocity decreases monotonously with the increase of the Marangoni number. Good agreements with space experimental data and most of previous numerical studies in the literature are evident. The terminal topological structure of flow field, in which a recirculation identical to Hill's vortex exists inside the drop, does not change with the Marangoni number. Only slight movement of the location of vortex center can be observed. On the contrary, bifurcations of the terminal topological structure of temperature field occur twice with increasing Marangoni number. At first, the uniform and straight layer-type structure of temperature field at infinitesimal Reynolds and Marangoni numbers wraps inside of the drop due to convective transport of heat as the Marangoni number increases, resulting in the emergence of an onion-type local cooler zone around the center of the drop beyond a lower critical Marangoni number. Expanding of this zone, particularly in the transverse direction, with the increasing of the Marangoni number leads to a cap- or even shell-type structure. The coldest point within the liquid drop locates on the axis. There is a middle critical Marangoni number, beyond which the coldest point will jump from the rear stagnation into the drop, though the topological structure of the temperature field does not change. The second bifurcation occurs at an upper critical Marangoni number, where the shell-type cooler zone inside drops ruptures from the central point and then a torus-type one emerges. The coldest point departs from the axis, and the socalled "cold-eye" appears in the meridian. It is also found that the inner and outer thermal boundary layers along the interface may exist both inside and outside the drop if $M a>70$. But the thickness decreases with the increasing Marangoni number more slowly than the prediction of potential flow at large Marangoni and Reynolds numbers. A velocity shear layer outside the drop is also introduced formally, of which modality may be affected by the convective transports of heat and/or momentum.
\end{abstract}

(ㄷ) 2011 Elsevier Ltd. All rights reserved.

\section{Introduction}

A fluid particle (gas bubble or liquid drop) floating in an immiscible bulk fluid with a temperature gradient can be moved by the non-uniform interface tension at the particle interface. This motion is well known as the thermocapillary or Marangoni migration. It plays an important role in many natural physical processes as well as a host of industrial activities, particularly in space material processing and many other scientific and engineering applications in microgravity, and attracts much more interests of researchers all over the world along with the progress of human space activities. The progress in this field has been summarized

\footnotetext{
* Corresponding author.

E-mail address: jfzhao@imech.ac.cn (J.-F. Zhao).
}

in the monograph by Subramanian and Balasubramaniam [1]. Thus, only studies relating to the thermocapillary migration of liquid drop are briefly reviewed here.

Thermocapillary migration was first analyzed by Young et al. [2] in the case of infinitesimal Reynolds and Marangoni numbers, in which convective transport of momentum and heat can be neglected comparing to molecular transport of these quantities and the governing equations can then be linearized. They derived the so-called YGB theory predicting the following steady migration velocity

$V_{\mathrm{YGB}}=\frac{2 U}{\left(2+3 \mu_{2} / \mu_{1}\right)\left(2+k_{2} / k_{1}\right)}$

where $U=-\sigma_{T} \nabla T_{\infty} R / \mu_{1}$ is the named thermocapillary velocity, $R$ is the drop radius, $\mu$ is the dynamic viscosity, $k$ is the thermal 


\section{Nomenclatures}

$\mathrm{Ca} \quad$ capillary number

$C_{p} \quad$ specific heat at constant pressure

$H \quad$ Heaviside function

$k \quad$ thermal conductivity

$\kappa$ interfacial curvature

n unit interface normal vector

$P \quad$ pressure

$\mathrm{Pr} \quad$ Prandtl number

$R \quad$ characteristic length

Re Reynolds number

u velocity vector

$u \quad r$-directional velocity

$v \quad z$-directional velocity

$U \quad$ characteristic velocity

$V \quad$ thermocapillary velocity

$V_{\text {YGB }} \quad$ YGB velocity

We Weber number

$T$ temperature

$\begin{array}{ll}\text { Greek symbols } \\ \varphi & \text { level set function } \\ \nabla T_{\infty} & \text { temperature gradient imposed } \\ \mu & \text { dynamic viscosity } \\ \rho & \text { density } \\ \sigma & \text { interface tension } \\ \sigma_{T} & \text { interface tension coefficient } \\ \alpha & \text { viscosity ratio } \\ \beta & \text { thermal conductivity ratio } \\ \chi & \text { specific heat ratio } \\ \xi & \text { density ratio } \\ \delta & \text { delta function } \\ \delta_{T} & \text { thickness of } T \text { boundary layer } \\ \delta_{V} & \text { thickness of } V \text { shear layer }\end{array}$

Subscripts

1 continuous phase

2 particle phase conductivity, $\sigma_{T}$ is the rate of change of interfacial tension with temperature, $\nabla T_{\infty}$ is the temperature gradient imposed in the continuous bulk fluid. The subscripts 1 and 2 denote the material properties of the continuous bulk fluid and the fluid particle, respectively. The Reynolds and Marangoni numbers are defined as $R e=U R / v_{1}$ and $M a=U R / \lambda_{1}$. Here, $v$ denotes the kinematic viscosity, $\lambda$ denotes the thermal diffusivity. If the Prandtl number is defined as $\operatorname{Pr}=v_{1} / \lambda_{1}$, one can obtain $M a=\operatorname{PrRe}$.

The analysis of Young et al. [2] was extended by many others to include convective influence. For example, using asymptotic expansion technique, the migration velocity of a nondeformable gas bubble for small but non-zero convective heat transfer in the limit of zero Reynolds number was obtained by Subramanian [3]. He found the migration speed of a gas bubble is reduced by the inclusion of the effect of convective transport of energy when $\mathrm{Ma}$ is small. In a later article, Subramanian [4] later extended this work to liquid drops, in these papers, they concluded that the heat convection may reduce the scaled migration velocity of the drop for small Ma. Balasubramaniam and Subramanian [5] studied the effect of the convection inside the drop and found the terminal velocity of a single drop first decreases with increasing Marangoni number, attains a minimum and then increases with a further increase in the Marangoni number.

On the other hand, numerical simulation was also used to help understanding the phenomenon. For example, Haj-Hariri et al. [6] calculated numerically the three-dimensional thermocapillary motion of deformable drops at finite Reynolds and Marangoni numbers and found that the strong heat convection may retard the thermocapillary motion of the drop. Ma et al. [7] analyzed the thermocapillary motion of a nondeformable single drop, and concluded that the scaled migration velocity decreases with $M a$, reaches a minimum, and then increases with $M a$ when $M a$ is large enough. Nas $[8,9]$ adopted the front-tracking method to calculate the thermocapillary interaction of two drops, Yin et al. [10] adopted the same method to numerically investigate the thermocapillary migration phenomena of a single nondeformable spherical drop. Brady et al. [11] recently presented a three-dimensional numerical simulation of the thermocapillary motion of a single deformable drop in a confined apparatus using a refined level-set grid method.

In addition to theoretical and numerical developments, there are some results from experiments in earth-based laboratories, as well as in reduced gravity conditions. Because of the non-linearity of the problem, the thermocapillary motion with finite values of the Reynolds and the Marangoni numbers can be observed experimentally only in microgravity environment in order to avoid the buoyant convection. Hadland et al. [12] and Xie et al. [13] reported some results on the thermocapillary migration of FC-75 droplets in silicone oil aboard a NASA space shuttle and the Chinese spaceship SZ-4, respectively. Both two experiments cover a wide range of the Reynolds and Marangoni numbers.

Using the same method as in our previous work [14] on gas bubbles, in which the level-set method is employed to catch the interface between the fluid particle and the continuous phase, a numerical study on the thermocapillary motion of deformable drops at moderate to large Marangoni number is presented in this paper. The results of the thermocapillary migration velocity will be compared with experimental data of Hadland et al. [12] and Xie et al. [13], as well as some previous numerical simulations reported by other researchers in the literature. The motivation of the present work, however, is to address the evolvement of the terminal topological structures of flow and temperature fields of a single deformable drop with the increasing Marangoni number in order to help understanding the characteristic of this phenomenon.

\section{Mathematical formulation and numerical method}

The thermocapillary migration of a single deformable drop in an immiscible bulk liquid is considered here with the following assumptions: (1) the fluids in both phases are Newtonian, viscous and incompressible; (2) the material properties are constant and not influenced by the temperature except the interface tension; (3) the interface tension depends linearly upon the temperature; and (4) the motion in both phases is axisymmetric and laminar.

To capture the interface of the drop, the level-set method is used. For details of the level set method, including the solve procedure and algorithm, please see our previous work [14]. Based on the level-set function and the continuum interface model, the dimensionless governing equations can be written as

$\nabla \cdot \mathbf{u}^{\prime}=0$

$$
\begin{aligned}
\frac{\partial \mathbf{u}^{\prime}}{\partial t^{\prime}}+\mathbf{u}^{\prime} \cdot \nabla \mathbf{u}^{\prime} & =-\frac{1}{\rho^{\prime}}\left\{\nabla p^{\prime}-\frac{\mu^{\prime} \nabla \cdot\left(\nabla \mathbf{u}^{\prime}+\nabla^{T} \mathbf{u}^{\prime}\right)}{\operatorname{Re}}\right. \\
+ & {\left.\left[\frac{1-C a\left(T-T_{0}\right)}{W e} \kappa \mathbf{n}+\frac{(\mathbf{I}-\mathbf{n n}) \cdot \nabla T}{\operatorname{Re}}\right] \delta(\varphi)\right\} }
\end{aligned}
$$


$\rho^{\prime} C_{p}^{\prime}\left(\frac{\partial T}{\partial t}+\mathbf{u}^{\prime} \cdot \nabla T\right)=\frac{1}{M a} \nabla \cdot\left(k^{\prime} \nabla T\right)$

here $R, U$, and $\left|\nabla T_{\infty}\right| R$ are used as the characteristic length, velocity and temperature, respectively. $T_{0}$ is a dimensionless reference temperature which is set as 0 or the value at the position of the center of the drop in the present study. The other dimensionless parameters appeared in the above equations are defined as follows

$\xi=\rho / \rho_{1}, \quad \alpha=\mu / \mu_{1}, \quad \chi=C_{p} / C_{p 1}, \quad \beta=k / k_{1}, \quad p^{\prime}=p / \rho_{1} U^{2}$,

$\mathrm{Ca}=\mu_{1} U / \sigma_{0}, \quad W e=\operatorname{ReCa}$,

The last two dimensionless parameters are the capillary and Weber numbers, which determine the deformation of the drop.

The unit interface normal vector, the interface curvature and the delta function which appeared in the above equations are also defined as follows

$\mathbf{n}=\nabla \varphi /|\nabla \varphi|$

$\kappa=\nabla \cdot \mathbf{n}$

$\delta(\varphi)= \begin{cases}1+\cos \left(\frac{2 \pi \varphi}{3 h}\right) /(3 h) & (|\varphi|<1.5 h) \\ 0 & (|\varphi| \geqslant 1.5 h)\end{cases}$

where $h$ is the grid spacing.

Furthermore, in order to avoid numerical instability caused by their jumps across the interface, the material properties of the fluids are smoothed by the Heaviside function

$\varsigma=\varsigma_{2}+\left(\varsigma_{1}-\varsigma_{2}\right) H$

where $\varsigma$ represents $\rho$, $\mu$, and so on. The Heaviside function $H$ is defined as

$H= \begin{cases}1 & (\varphi \geqslant 1.5 h) \\ \frac{1}{2}+\frac{\varphi}{3 h}+\sin \left(\frac{2 \pi \varphi}{3 h}\right) / 2 \pi & (|\varphi|<1.5 h) \\ 0 & (\varphi \leqslant-1.5 h)\end{cases}$

The projection [15] method is used to solve the above governing equations with uniform staggered grid of equal spacing. The following initial conditions

$u=v=0, \quad T=z \quad(t=0)$

and the boundary conditions:

$$
\left\{\begin{array}{lll}
u=v=0, & T=-A & (z=-A) \\
u=v=0, & T=B & (z=B) \\
u=\frac{\partial v}{\partial r}=\frac{\partial T}{\partial r}=0 & & (r=0) \\
u=v=0, & T=z & (r=C)
\end{array}\right.
$$

are used, where $z=-A, z=B$, and $r=C$ denote the bottom, top, and the outer boundaries, respectively. The center of the drop locates at the point $(0,0)$ at the beginning, and $A$ is always set as 3 .

The computational domain was chosen to be $15 \times 5$, while a grid number of $300 \times 100$ is used. Comparisons between the numerical prediction of the terminal velocity of a drop at and that predicted by the YGB theory show that the motion of the drop is not affected by the computational boundary, and that the computational precision can be guaranteed.

\section{Compare with space experimental data}

The thermocapillary motion of deformable FC-75 drops in silicone oil is studied numerically using the above algorithm at the conditions corresponding to those in the space experiments of Hadland et al. [12] and Xie et al. [13]. Constant material properties, however, are assumed to be independent of temperature. The fol- lowing values are adopted for the dimensionless parameters in the problem, namely $\xi=1.89, \alpha=0.14, \beta=0.47, \chi=0.69$, and $\operatorname{Pr}=83.3$. Furthermore, a slightly larger value for the capillary number, i.e. $\mathrm{Ca}=0.2$ which is still much less than 1 to guarantee no distinct deformation of the drop, is used here than those in the space experiments, which are of the order of $10^{-1}$ or less, in order to prevent the virtual flow caused by the strong jump of the normal stress across the interface of drops.

Fig. 1 shows evolutions of drop migration velocities at different Marangoni numbers. It ought to be pointed out before further discussions that the fall of the scaled velocities in the marked range is due to the influence of the upper wall on the flow and temperature fields. The positions of the drop center at $z=8$ are also shown in the figure to indicate this influence. It is clearly shown that this influence will not be evident unless the dimensionless distance between the drop and the upper wall is less than about 4. Similar conclusion can also be found in Subramanian and Balasubramaniam [1]. Thus, a reasonable terminating distance ought to be adopted.

The early transients of the motion, caused by the preternatural initial conditions used in the numerical simulations, show the same characteristics as those reported by Haj-Hariri et al. [6], Nas [8,9], Yin et al. [10], and Brady et al. [11]. Here, however, we are mainly concerned with the terminal state of the thermocapillary motion. It is evident that a steady terminal state can always be reached, which can also be observed in the three-dimensional numerical simulations of Brady et al. [11] with constant material properties. The predicted dimensionless terminal velocity is a monotonically decreasing function of the Marangoni number at steady state. Fig. 2 shows the steady terminal migration velocities scaled using the corresponding values predicted by the YGB theory. The experimental data of Hadland et al. [12] and Xie et al. [13], labeled respectively as HBWS-1999 and XHZLH-2005 for the brevity of the figure are also shown in the same figure for comparison. Generally, good agreements are evident.

Some previous numerical results, such as Haj-Hariri et al. [6], Ma et al. [7] and Yin et al. [10], labeled respectively as HHSB1997, Ma-1998 and YGHC-2008, are also shown in Fig. 2. Except the prediction of Ma et al. [7], the other numerical simulations including the present work all show that the predicted dimensionless terminal velocity is a monotonically decreasing function of the Marangoni number. The differences among the present results and those of Haj-Hariri et al. [6] and Yin et al. [10] may be caused by the different values of material parameters used in the calculations. For example, Haj-Hariri et al. [6] used $\xi=\alpha=\beta=\chi=0.5$, and $C a=0.1$, while Yin et al. [10] used $\xi=\alpha=\beta=\chi=1$, and nonde-

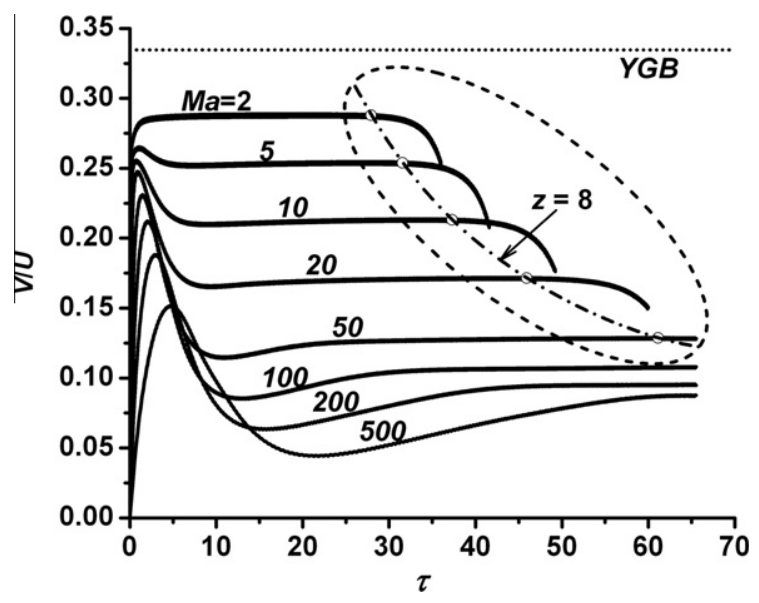

Fig. 1. Evolutions of the scaled migration velocity versus the dimensionless time. 


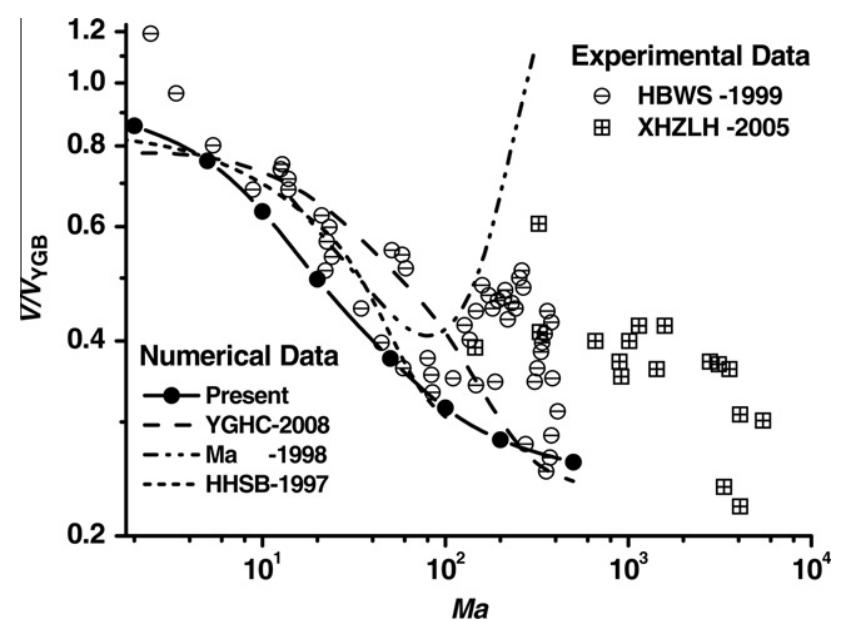

Fig. 2. Comparison of the predicted of the steady terminal migration velocity with space experimental data and other numerical data reported in the literature.

formable drop. The terminal migration velocities can be affected by the change of material parameters. Thus, the differences are reasonable and may be diminished if these influences are taken into account.

Some typical streamlines of flow field at the steady terminal state are plotted in Fig. 3, in which the left part is those in the local reference frame attached to the center of the drop and the right part is those in the laboratory reference frame. The terminal topological structure of flow field, in which a recirculation identical to
Hill's vortex exists inside the drop, does not change with the Marangoni number. The center of the vortex locates near the interface of the drop. As shown in Fig. 4, the transverse position of the vortex in the local reference frame is not changed with the Marangoni number, however, in the laboratory reference frame it moves outward with the Marangoni number; the longitudinal positions in both two reference frames move downstream with the increase of the Marangoni number. The reason for this fact should be found in the distribution of temperature. The temperature gradient is nearly uniform along the drop interface at small Marangoni number, while a much uneven distribution of temperature will be observed at high Marangoni number, these can be seen in Fig. 5. Although the interface temperature gradient over whole interface decreases at high Marangoni number, it becomes larger along the rear part than that along the front part. Larger temperature gradient means larger driving force and faster motion, so the center of the vortex inside drops moves with the increase of the Marangoni number.

Fig. 5 provides an evolution process of the isotherm with increasing Marangoni number. The right part of each figure shows isotherms of the temperature, while the left one shows those of the perturbed temperature, namely the difference between the terminal temperature and the initial temperature at the same position. And the isotherms of the terminal temperature field are also shown in the right part. An equal dimensionless temperature increment of 0.5 is adopted for all isotherms. Obviously, the enhanced convective transport of momentum and heat with the increase of the Marangoni number results in the wrapping of the isotherms toward the rear of the drop, leading to a substantial reduction in the interface temperature gradient and diminishing

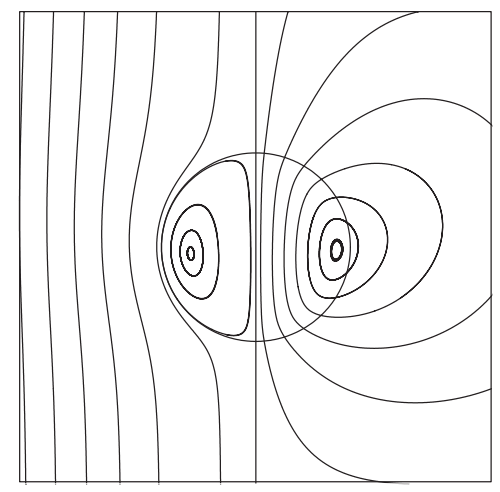

(a) $M a=5$

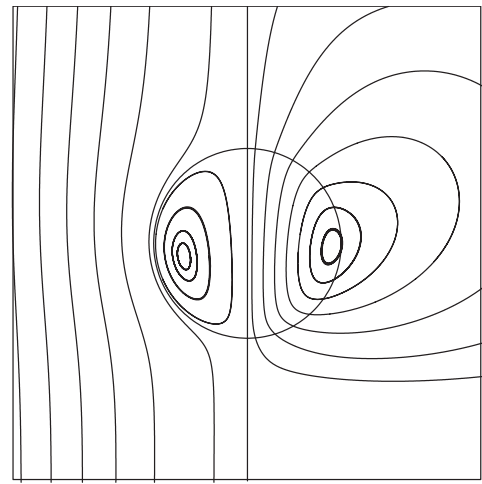

(c) $M a=50$

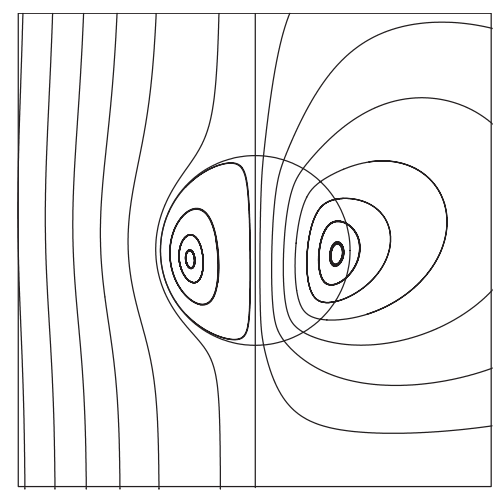

(b) $M a=10$

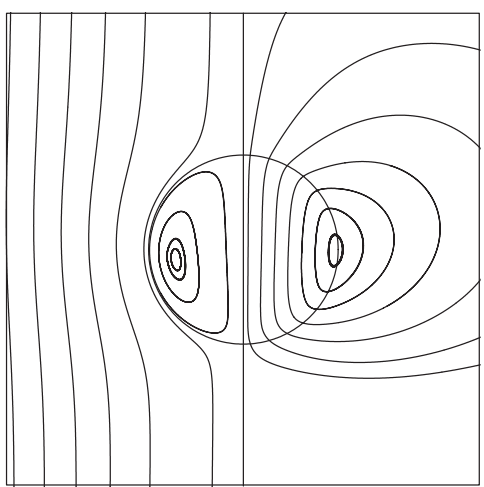

(d) $M a=300$

Fig. 3. Streamlines at different Marangoni numbers. 


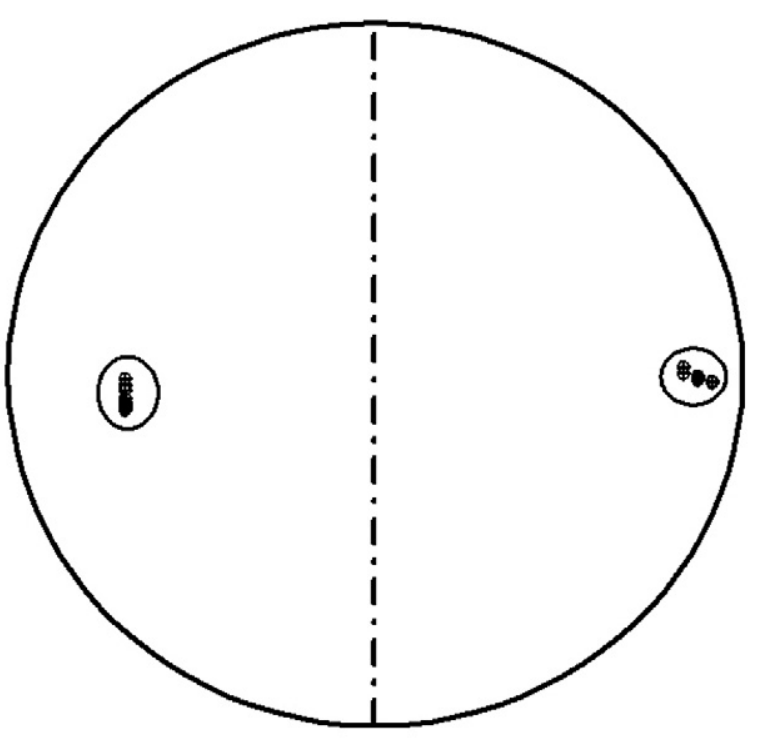

(a) whole chart

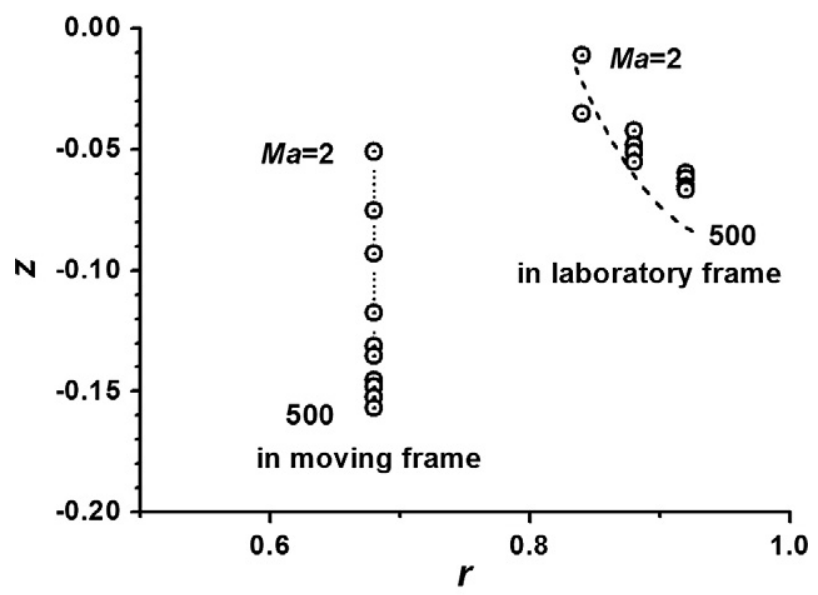

(b) part enlarged chart

Fig. 4. The locations of the center of the vortex inside drops relative to the center of drops. (Left: local reference frame. Right: laboratory reference frame.)

the driving force for the motion of the drop. That is the reason of the fact that the migration velocity decreases monotonically with the increase of the Marangoni number.

According to the linear YGB theory, a uniform and straight layer-type structure of temperature field exists at infinitesimal Reynolds and Marangoni numbers. The coldest point locates on the rear stagnation, namely the bottom intersection between the drop's axis and its interface. At small but finite Marangoni number, wrapping of isotherms caused by the convective transport of heat results in a distorted layer-type structure and negative perturbed isotherm appearing (Fig. 5a). Further increasing of the Marangoni number will lead the emergence of an onion-type local cooler zone around the center of the drop, as shown in Fig. 5b. This is the first bifurcation of the topological structure of the terminal temperature field, and the corresponding value of the Marangoni number is then called as the lower critical Marangoni number. The coldest point, however, still locates on the rear stagnation at first. Expanding of this zone, particularly in the transverse direction, with the increasing of the Marangoni number leads to a cap-type structure (Fig. 5c). This cooler zone also moves upwards with the increasing Marangoni number. There is a middle critical Marangoni number, beyond which the coldest point will jump from the rear stagnation into the drop, though the topological structure of the temperature field does not change. Perturbed temperature field also expands bidirectionally along the axis, especially downward to the rear of the drop due to the strengthening convective transport.

With further increasing Marangoni number, the cap-type structure of the local cooler zone moves upwards with a transverse expanding and a longitudinal shrinking, leading to a shell-type one. The center part of the shell-type cooler zone inside drops will be thinned and thinned. It will finally rupture from the central point and then form a torus-type one (Fig. 5d). It is the second bifurcation of the topological structure of steady terminal temperature field, and thus the upper critical Marangoni number is introduced here. Beyond it, the coldest point split departs from the axis to form a coldest line, and the so-called "cold-eye" appears in the meridian. On the contrary, no change occurs in the topological structure of the perturbed temperature field, and the negative zone continues to expand downwards, but its center part shrinks adversely. Slight transverse expanding of the negative zone also occurs with the increasing Marangoni number, resulting in the emergence of a shell-type structure, similar to the terminal temperature field. Thus, the second bifurcation of the topological structure of terminal perturbed temperature field may also occur at a much larger Marangoni number.

The above thermal structures have also been obtained in the previous numerical simulations, but no allegation on the evolution process has been made in the literature. It is observed that the evolution of the topological structure of temperature field reported here is consistent with that in Haj-Hariri et al. [6] in the same range of the Marangoni number. However, a relatively slower evolution was found in Yin et al. [10], while a much quicker one in Ma et al. [7]. In the latter work, the second bifurcation of the topological structure of temperature field occurs at a much smaller Marangoni number, and then the thermal structure at $M a=100$ (shown in Fig. 9 of Ma et al. [7]) is close to that at $M a \approx 500$ in the present work. Thus, much quicker decrease of the thickness of thermal boundary layers both inside and outside the drop can be observed in Ma et al. [7]. Although it is not clear for the reason of the differences, the validity of the present results may be guaranteed by the agreements with other numerical simulations as well as experimental data.

The movement of the coldest point within the drop at different Marangoni number is shown in Fig. 6 . If $M a \leqslant 11$, the coldest point within the drop locates on the rear stagnation. If $M a \geqslant 12$, it will jump into the drop, even locate at a position above the center of the drop. Then it moves upwards along the axis due to wrapping of isotherms caused by the enhanced convective transport of heat. As the torus-type local cooler zone is formed, the coldest line, not a sole point, within the drop will depart from the axis of the drop. According to the above analysis, the middle critical Marangoni number in our present condition may be located in the range from 11 to 12 , while the lower and upper critical ones are approximately 10 and 100 , respectively.

Fig. 7 shows the aspect ratio of the longitudinal length over the transverse one of the deformed drop at the terminal steady state of the thermocapillary migration. Those for bubbles obtained in our previous work [14] are also shown for comparison. A larger deformation of a bubble can be observed than that of a drop at the same Marangoni number. The aspect ratio of drop increases quickly with the increase of the Marangoni number. The biggest variation of aspect ratio, however, is no more than $1 \%$ in the present study, because both the Reynolds and capillary numbers are very small. It ought to be pointed out here that the actual deformation may be much smaller than the predictions since the capillary number in space experiments has smaller values than that used here. Therefore, no distinct influence of the deformation of drops can occur 


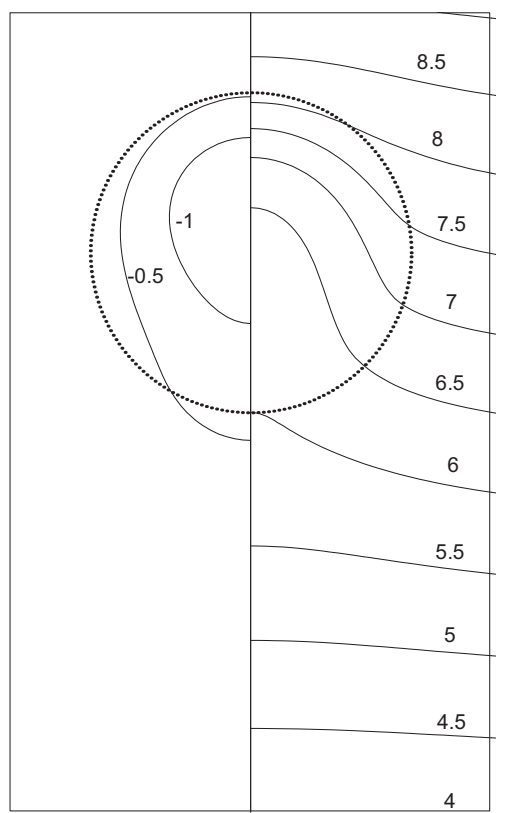

(a) $M a=5$

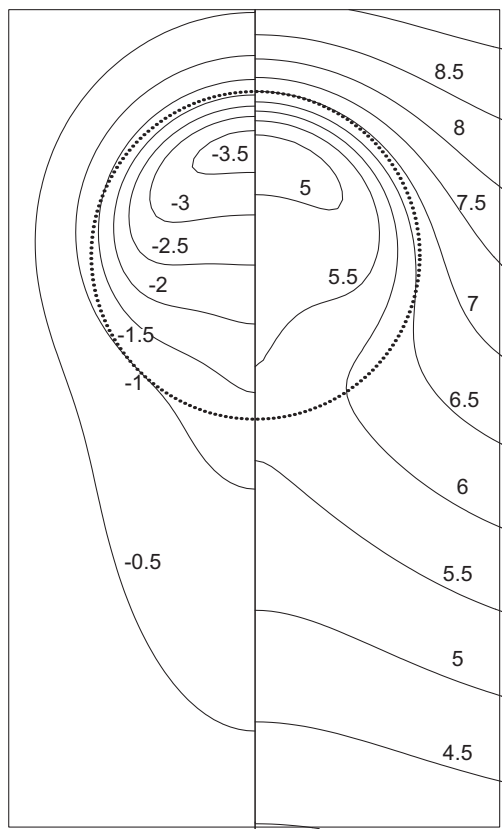

(c) $M a=50$

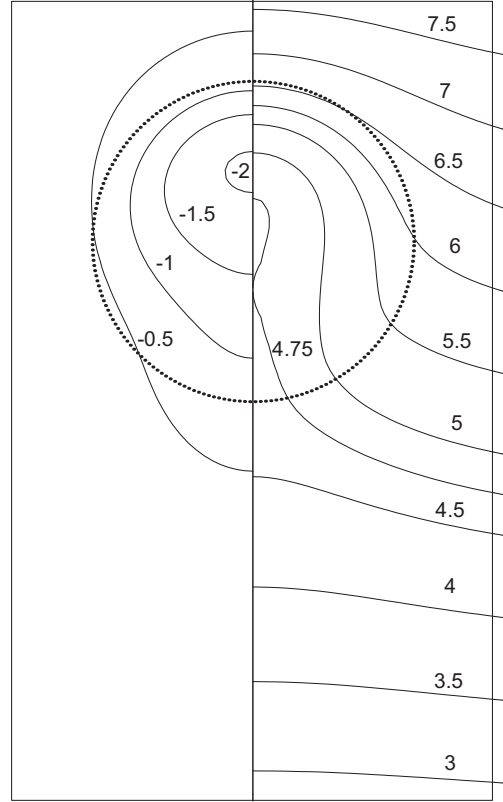

(b) $M a=10$

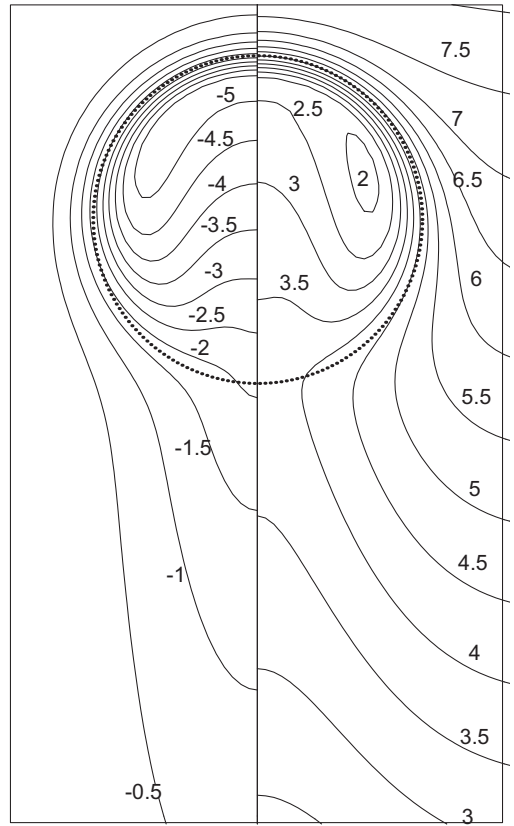

(d) $M a=300$

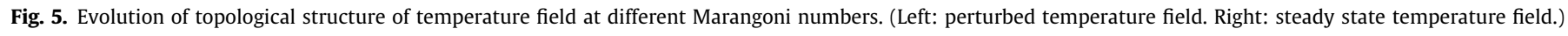

in the thermocapillary migration, at least within the present parameter ranges.

Fig. 8 shows the variances of the temperature and its radial gradient along a radial direction starting from the drop's center at an angle of $\theta=60^{\circ}$ with regard to the moving direction of the drop. If the inner and outer thermal boundary layers attached to the interface are introduced formally in normal sense, their thickness can be defined straightforwardly. The big jump of the gradient between $r=0.9$ and $r=1.1$ is shown here by the short dotted line due to the physical discontinuity across the interface. There exists a difficulty to find reasonable values of the inside and outside temperature gradients on the interface, which is caused by the continuum interface model. Thus, the difference of the maximum and the fixed gradient far away from the drop is adopted as the reference for the outer thermal boundary layer. The boundary is defined at such a position where the radial gradient of the temperature is equal to $1 \%$ of the reference above the fixed gradient far away from the drop. The point of zero gradient is simply used as the boundary of the inner thermal boundary layer.

As shown in Fig. 9, both thicknesses of the inner and outer thermal boundary layers increase with the increasing angle at two Marangoni numbers. The thickness of the inner thermal boundary 


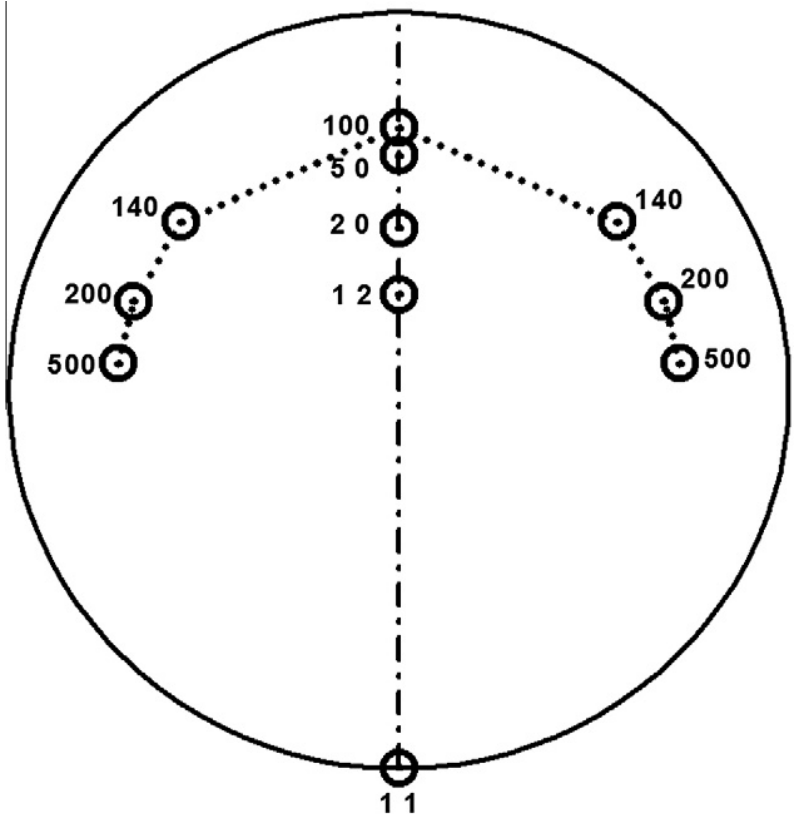

Fig. 6. The coldest position inside drops.

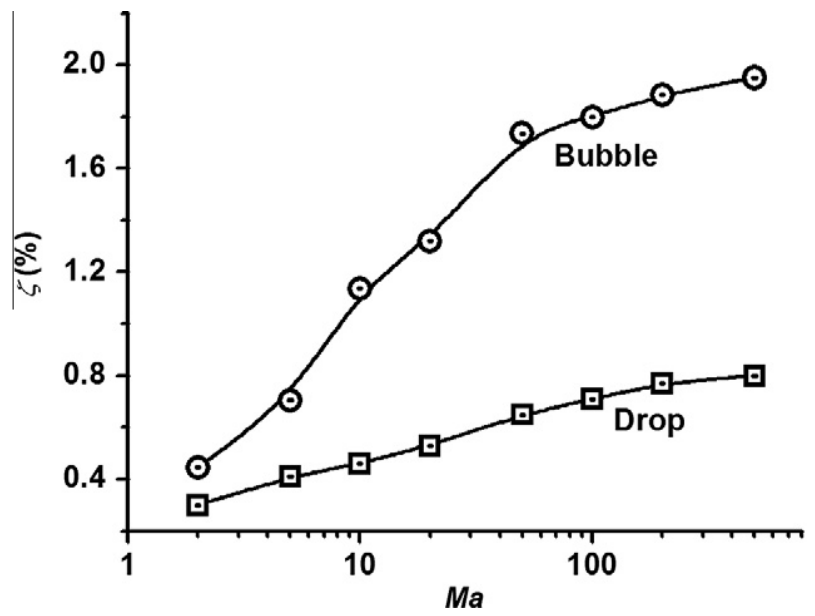

Fig. 7. Aspect ratio of the deformable drop and bubble at different Marangoni numbers.

layer increases slowly and keeps the magnitude of $O\left(10^{-1}\right)$ before some angle, while beyond this angle no thermal boundary layer, even formally, can be observed. The thickness of the outer thermal boundary layer keeps constant approximate to a steady value of $O\left(10^{\circ}\right)$ before an angle of about $110^{\circ}$, and increases sharply with further increasing angle. Therefore, the thickness at the angle of $60^{\circ}$ can be used to characterize the inner and outer thermal boundary layer, as shown in Fig. 10. A separatrix is shown as dashed lines. There is no doubt that the inner and outer thermal boundary layers exist in the common meaning if $M a>70$. The thickness of the outer thermal boundary layer varies as $\delta_{T} \sim O\left(\mathrm{Ma}^{-2 / 3}\right)$ in this range, while a negative exponent much close to 0 can be found for the inner one. A potential theory, however, of $\delta_{T} \sim O\left(M a^{-1}\right)$ was obtained for both inner and outer thermal boundary layers by Balasubramaniam and Subramanian [5]. There exists much a large difference between our result and the potential theory, which can be explained as follows. The maximum Reynolds number in the present work is about 6 , which is not large enough to preserve a potential flow in both phases. Furthermore, the short dotted line

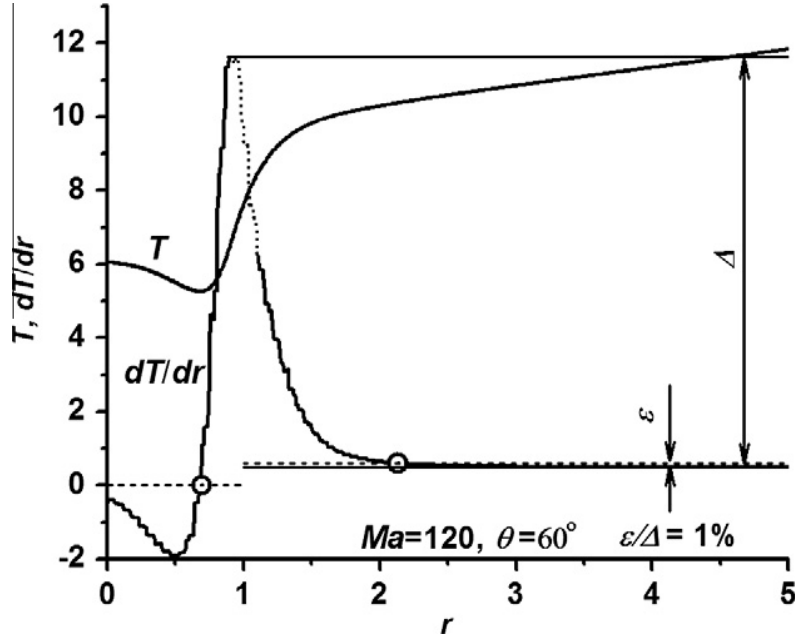

Fig. 8. Definition of the inner and outer thermal boundary layer $\left(M a=120, \theta=60^{\circ}\right)$.

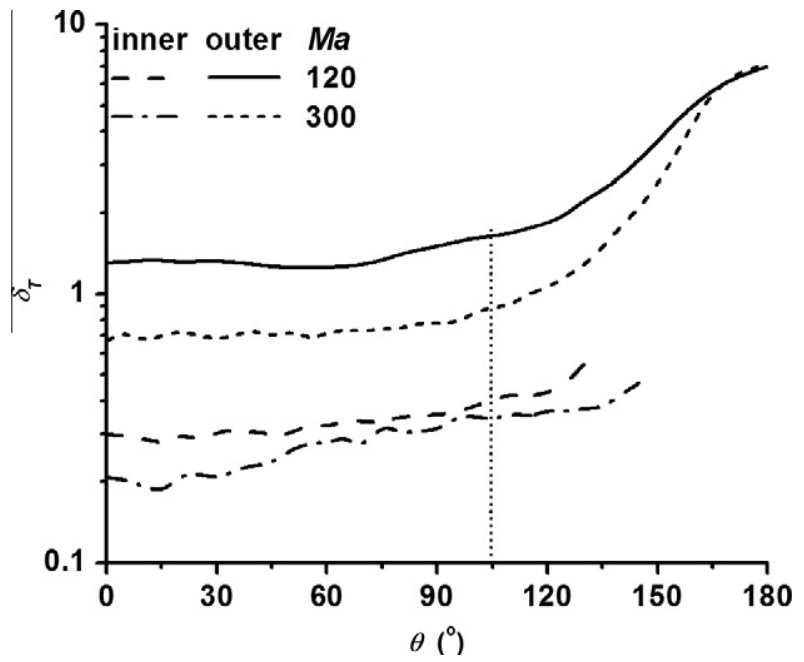

Fig. 9. Thickness of inner and outer thermal boundary layer at different angle $(M a=120$ and 300).

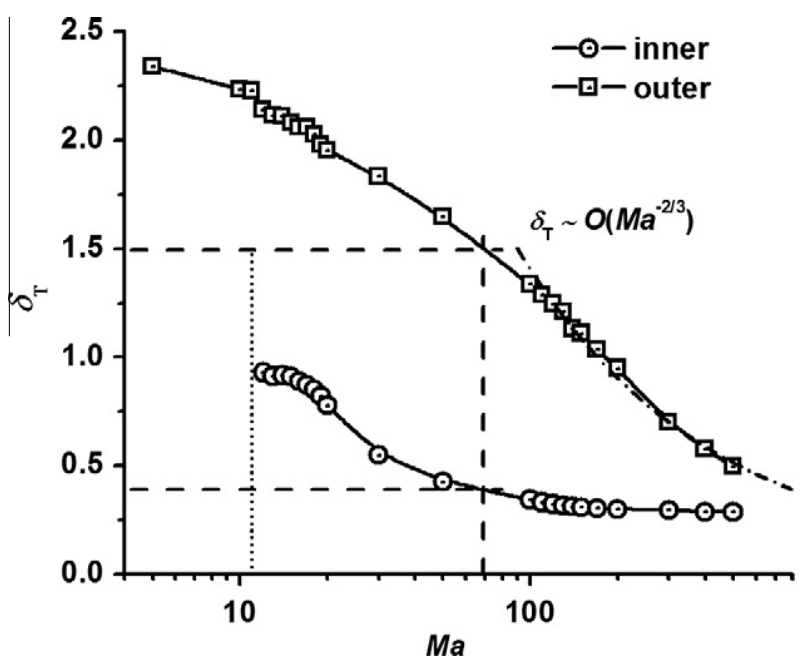

Fig. 10. Thicknesses of the inner and outer thermal boundary layers at different Marangoni number. 


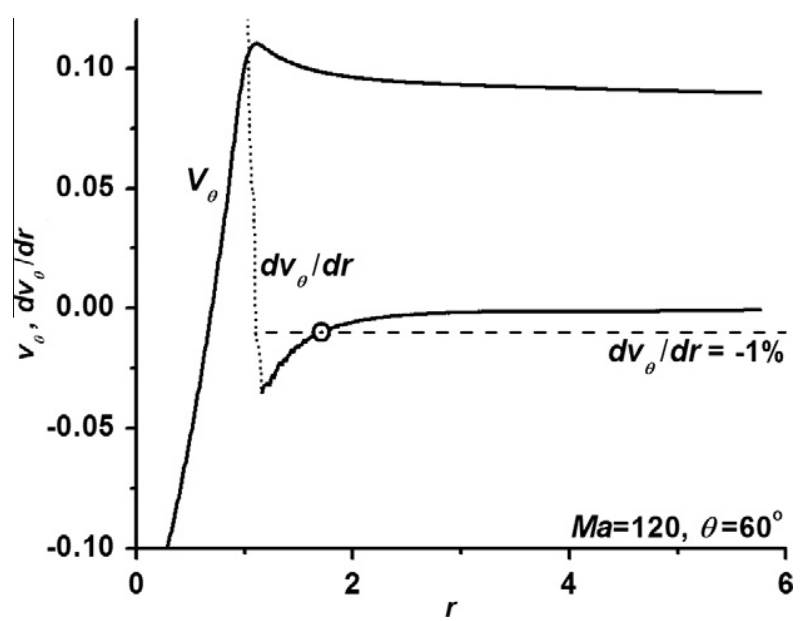

Fig. 11. Definition of the shear layer $\left(M a=120, \theta=60^{\circ}\right)$.

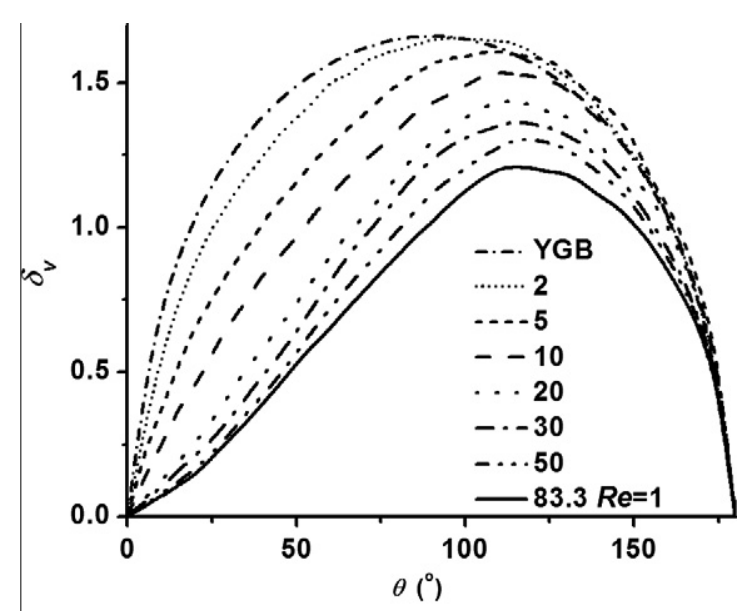

(a) $\operatorname{Re}<1$

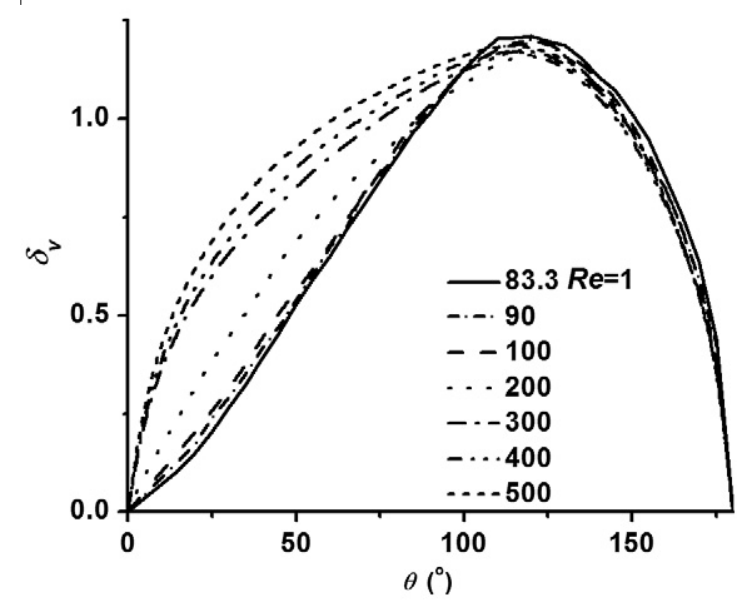

(b) $\operatorname{Re}>1$

Fig. 12. Circumferential distribution of the shear layer thickness at different Marangoni number.

shown in Fig. 10 indicates that no inner boundary layer exists even in formal if $M a<12$.

Typical variances of the tangential velocity and its radial gradients along the radial direction are shown in Fig. 11. A big jump of the radial gradient of tangential velocity also exists in the range from $r=0.9$ to $r=1.1$, which is shown here by the short dotted line.

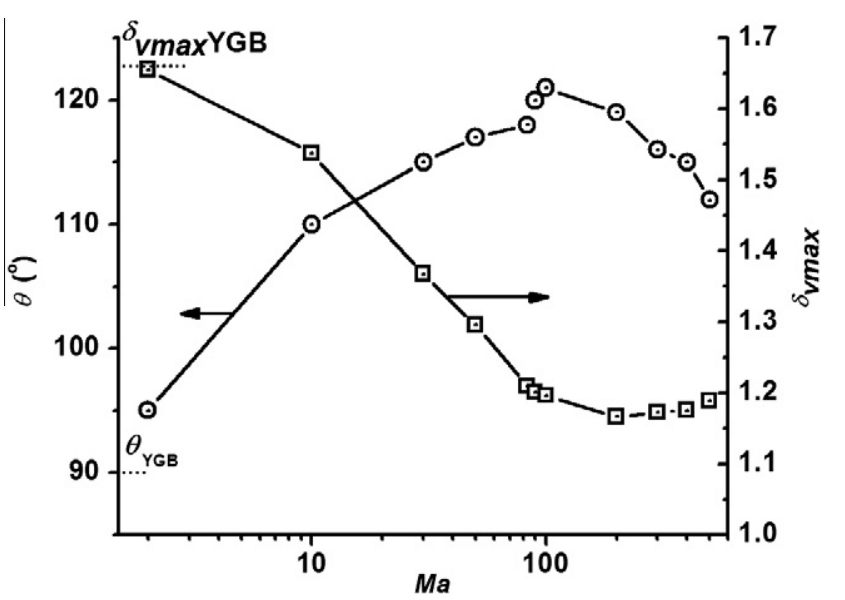

Fig. 13. The maximum of the shear layer thickness and its corresponding position at different Marangoni number.

Large value of the gradient throughout the whole range inside the drop, then strong shear effect exists everywhere inside the drop. Outside the drop, the radial gradient of tangential velocity has a negative value. The maximum of its absolute value occurs on the interface. Because of small Reynolds number in the whole field, there is no classical boundary layer outside of the drop. A layer abutting the drop's interface, however, with distinct greater values of radial gradient of tangential velocity can be observed, which is called as the shear layer here. The boundary of the shear layer is defined at such a position where the absolute gradient is equal to $1 \%$ of this maximum. In addition, a value of zero is set for the angle of both $0^{\circ}$ and $180^{\circ}$ due to the axisymmetric assumption.

As shown in Fig. 12a, a good agreement of the present results of the circumferential distribution of the shear layer thickness with YGB theory is evident at small Marangoni number. With the increasing Marangoni number, the configuration of the circumferential distribution of the shear layer thickness will be declined downstream at first (Fig. 12a). The maximum thickness decreases and its position moves simultaneity downwards. On the contrary, further increasing of the Marangoni number after $M a>83.3$ can cause only a slight decrease, or even slight increase, of the maximum thickness of the shear layer, while its position moves upwards instead (Fig. 12b). Furthermore, the first half part the configuration of the circumferential distribution of the shear layer thickness expands instead of shrinks. A weak jump of the position of the maximum thickness occurs across $M a=83.3$ (Fig. 13), which is corresponding to $R e=1$ because of $P r=83.3$ in the present work. The reason for the different behaviors mentioned above may lie on the following fact. If $R e<1$, or $M a<83.3$, the flow field will be dominated by the convective transport of heat and the diffusive transport of momentum, while the convective transports of both heat and momentum dominate the flow field if $\operatorname{Re}>1$, or $M a>83.3$.

\section{Conclusions}

The thermocapillary migration of deformable FC-75 drops in silicone oil has been studied numerically up to $M a=500$. The axisymmetric governing equations are solved using the projection method with a uniform staggered grid of equal spacing at appropriate initial and boundary conditions. The level-set method is used to account for finite drop deformation, and the continuum interface model is also adopted to model the interface behaviors. Conditions corresponding to those in the space experiments of Hadland et al. [12] and Xie et al. [13] are used, while constant material properties are assumed to be independent of temperature except the interface 
tension. Steady terminal state of the motion can always be reached. The dimensionless terminal migration velocity decreases monotonously with the increase of the Marangoni number. Good agreements with space experimental data and most of previous numerical studies in the literature are evident.

Evolvement of the topological structures of the terminal flow and temperature fields are analyzed in detail. The terminal topological structure of flow field, in which a recirculation identical to Hill's vortex exists inside the drop, does not change with the Marangoni number. Only slight movement of the location of vortex center can be observed. On the contrary, bifurcations of the terminal topological structure of temperature field occur twice with increasing Marangoni number. At first, the uniform and straight layer-type structure of temperature field at infinitesimal Reynolds and Marangoni numbers wraps inside of the drop due to convective transport of heat as the Marangoni number increases, resulting in the emergence of a onion-type local cooler zone around the center of the drop beyond a lower critical Marangoni number. Expanding of this zone, particularly in the transverse direction, with the increasing of the Marangoni number leads to a cap- or even shell-type structure. The coldest point within the liquid drop locates on the axis. There is a middle critical Marangoni number, beyond which the coldest point will jump from the rear stagnation into the drop, though the topological structure of the temperature field does not change. The second bifurcation occurs at an upper critical Marangoni number, where the shell-type cooler zone inside drops ruptures from the central point and then a torus-type one emerges. The coldest point departs from the axis to form the coldest circle, and the so-called "cold-eye" appears in the meridian.

The inner and outer thermal boundary layers along the interface may exist both inside and outside the drop if $M a>70$. But the thickness decreases with the increasing Marangoni number more slowly than the prediction of potential flow at large Marangoni and Reynolds numbers. A shear layer outside the drop is also introduced formally, of which modality may be affected by the modes of heat and momentum transports.

\section{Acknowledgment}

The present work is supported financially by the National Natural Science Foundation of China under the grant of 10972225.

\section{References}

[1] R.S. Subramanian, R. Balasubramaniam, The Motion of Bubbles and Drops in Reduced Gravity, Cambridge University Press, Cambridge, UK, 2001.

[2] N.O. Young, J.S. Goldstein, M.J. Block, The motion of bubbles in a vertical temperature gradient, J. Fluid Mech. 6 (1959) 350-356.

[3] R.S. Subramanian, Slow migration of a gas bubble in a thermal gradient, AIChE J. 27 (4) (1981) 646-654.

[4] R.S. Subramanian, Thermocapillary migration of bubbles and droplets, Adv. Space Res. 3 (5) (1983) 145-153.

[5] R. Balasubramaniam, R.S. Subramanian, The migration of a drop in a uniform temperature gradient at large Marangoni numbers, Phys. Fluids 12 (4) (2000) 733-743.

[6] H. Haj-Hariri, Q. Shi, A. Borhan, Thermocapillary motion of deformable drops at finite Reynolds and Marangoni numbers, Phys. Fluids 9 (4) (1997) 845-855.

[7] X.J. Ma, R. Balasubramaniam, R.S. Subramanian, Numerical simulation of thermocapillary drop motion with internal circulation, Numer. Heat Transfer A - Appl. 35 (1999) 291-309.

[8] S. Nas, Computational investigation of thermocapillary migration of bubbles and drops in zero gravity, Ph.D. Thesis, Department of Aerospace Engineering, University of Michigan, USA, 1995.

[9] S. Nas, G. Tryggvason, Thermocapillary interaction of two bubbles or drops, Int. J. Multiphase Flow 29 (2003) 1117-1135.

[10] Z. Yin, P. Gao, W. Hu, L. Chang, Thermocapillary migration of nondeformable drops, Phys. Fluids 20 (2008) 082101.

[11] P.T. Brady, M. Herrmann, J.M. Lopez, Confined thermocapillary motion of a three-dimensional deformable drop, Phys. Fluids 23 (2011) 022101.

[12] P.H. Hadland, R. Balasubramaniam, G. Wozniak, R.S. Subramanian, Thermocapillary migration of bubbles and drops at moderate to large Marangoni number and moderate Reynolds number in reduced gravity, Exp. Fluids 26 (2009) 240-248.

[13] J.C. Xie, H. Lin, P. Zhang, F. Liu, W.R. Hu, Experimental investigation on thermocapillary drop migration at large Marangoni number in reduced gravity, J. Colloid Interface Sci. 285 (2005) 737-743.

[14] J.Fu. Zhao, Zh.D. Li, H.X. Li, J. Li, Thermocapillary migration of deformable drops at moderate to large Marangoni number in microgravity, Microgravity Sci. Technol. 22 (3) (2010) 295-303.

[15] G. Son, V.K. Dhir, Numerical simulation of film boiling near critical pressures with a level set method, J. Heat Transfer 120 (1998) 183-192. 